UTTG-12-02

\title{
Adiabatic Modes in Cosmology
}

\author{
Steven Weinberg ${ }^{1}$ \\ Theory Group, Department of Physics, University of Texas \\ Austin, TX, 78712
}

\begin{abstract}
We show that the field equations for cosmological perturbations in Newtonian gauge always have an adiabatic solution, for which a quantity $\mathcal{R}$ is non-zero and constant in all eras in the limit of large wavelength, so that it can be used to connect observed cosmological fluctuations in this mode with those at very early times. There is also a second adiabatic mode, for which $\mathcal{R}$ vanishes for large wavelength, and in general there may be non-adiabatic modes as well. These conclusions apply in all eras and whatever the constituents of the universe, under only a mild technical assumption about the wavelength dependence of the field equations for large wave length. In the absence of anisotropic inertia, the perturbations in the adiabatic modes are given for large wavelength by universal formulas in terms of the Robertson-Walker scale factor. We discuss an apparent discrepancy between these results and what appears to be a conservation law in all modes found for large wavelength in synchronous gauge: it turns out that, although equivalent, synchronous and Newtonian gauges suggest inequivalent assumptions about the behavior of the perturbations for large wavelength.
\end{abstract}

\footnotetext{
${ }^{1}$ Electronic address: weinberg@physics.utexas.edu
} 


\section{INTRODUCTION}

If observations are to be used to tell us something about inflation, then we need some way of connecting the properties of the cosmological fluctuations produced during inflation to the properties of fluctuations much closer to the present. Inconveniently, in inflationary cosmologies the era of inflation was followed by a period when the energy in scalar fields was converted into matter and radiation, and about this process we know essentially nothing. Subsequently there may have been other periods about which we are equally ignorant, such as the often-hypothesized era with temperatures between $10^{13}$ $\mathrm{GeV}$ and $10^{11} \mathrm{GeV}$, when supersymmetry may have become broken by unknown strong forces. These mysterious eras occurred when fluctuations of cosmological interest were far outside the horizon, but this does not rule out some effect on the strength or even the wave-length dependence of these fluctuations. ${ }^{2}$ Therefore, in relating the cosmological fluctuations produced during inflation with those observed in the cosmic microwave background or in large-scale cosmic structures, it is essential to employ some sort of conservation law that is valid at large wavelengths whatever the details of cosmic evolution.

In much work on fluctuations in cosmology, the conserved quantity is taken to be a quantity $\mathcal{R}$ related to the spatial curvature on co-moving spatial surfaces[1], given in Newtonian gauge by ${ }^{3}$

$$
\mathcal{R}=-\Psi+H \delta u \text {. }
$$

The rate of change of $\mathcal{R}$ is given by a general formula[2]:

$$
\dot{\mathcal{R}}=X+\left(\frac{q^{2}}{a^{2}}\right)\left[\left(\frac{\ddot{H}+3 H \dot{H}}{3 \dot{H}^{2}}\right) \Psi-\left(\frac{H}{\dot{H}}\right) 4 \pi G \delta \sigma\right] .
$$

\footnotetext{
${ }^{2}$ By a mode being "beyond the horizon" we only mean that the physical wave number is much less than the expansion rate. This does not necessarily have anything to do with causality; indeed, the point of inflation is to make the true particle horizon radius much larger than the inverse expansion rate.

${ }^{3}$ Here $H=\dot{a} / a$ is the expansion rate, with dots denoting ordinary time derivatives. In Newtonian gauge the perturbations to the gravitational field are taken to be $\delta g_{00}=-2 \Phi$ and $\delta g_{i j}=-2 a^{2} \Psi \delta_{i j}$. Also $\delta \rho, \delta p$, and $\delta u$ are the perturbations to the total energy density, pressure, and velocity potential in Newtonian gauge, while we use a bar to denote unperturbed quantities like the unperturbed energy density $\bar{\rho}$ and pressure $\bar{p}$. For simplicity we assume a vanishing unperturbed spatial curvature.
} 
Here $q$ is the co-moving wave number, $\delta \sigma$ is a measure of the anisotropic stress, ${ }^{4}$ and

$$
X \equiv \frac{\dot{\bar{\rho}} \delta p-\dot{\bar{p}} \delta \rho}{3(\bar{\rho}+\bar{p})^{2}} .
$$

Thus $\mathcal{R}$ is conserved in the limit of small wave number if and only if $X=0$ in this limit.

The limit of small $q$ is of some interest in itself, but its importance lies chiefly in the circumstance that those factors of $q$ that arise from the field equations (rather than from the initial conditions) are always accompanied by factors of $1 / a(t)$, because it is only $q / a(t)$ that is independent of the units chosen for the co-moving spatial coordinates $x^{i}$. It is usually a good guess that terms in the perturbations proportional to such factors of $q$ will be negligible if $q / a(t) H(t) \ll 1$. Hence, although here we will study the behavior of the perturbations in the limit of small $q$, it is expected that this provides insight to the behavior of perturbations as $a(t)$ increases. In particular, we expect that if $X \rightarrow 0$ for $q \rightarrow 0$, and if the coefficient of $q^{2}$ in the second term in Eq. (2) remains finite in this limit, then at any given epoch $\dot{\mathcal{R}}$ will be small if $q / a H$ is sufficiently small. In any mode for which $\mathcal{R}$ is non-zero in this limit, the fractional rate of change of $\mathcal{R}$ will then also be small in this limit.

Now, the quantity $X$ vanishes in all modes and for all $q$ when the perturbed pressure $\bar{p}+\delta p$ is a function only of the perturbed energy density $\bar{\rho}+\delta \rho$, as is the case in a universe dominated either by pure radiation or by pure cold matter, but not when both radiation and cold matter are important, and also not during inflation or in the curvaton model[4]. The quantity $X$ does vanish for all modes in the limit $q \rightarrow 0$ in the case of inflation with a single scalar field, but this is not true with several scalar fields. Section II of this paper will show that in general, whatever the contents of the universe, with only a mild technical assumption about the dependence on wave number of the field equations for cosmological perturbations in Newtonian gauge, these equations always have a physical solution for which $X \rightarrow 0$ and $\mathcal{R}$ approaches a non-zero constant in the limit $q \rightarrow 0$, though there may also be other modes for which $\mathcal{R}$ is not constant. In fact, there are always at least

\footnotetext{
${ }^{4}$ The quantity $\delta \sigma$ is defined by writing $T_{i j}$ for scalar perturbations as $g_{i j} p+\partial_{i} \partial_{j} \delta \sigma$. In this formalism, $p$ includes effects of bulk viscosity, while $i \mathbf{q} \delta u$ is the velocity of energy transport, and so includes effects of heat conduction. For this formalism, see ref. [3].
} 
two solutions with $X=0$ and $\mathcal{R}$ constant in the limit $q \rightarrow 0$ : one solution for which $\mathcal{R} \neq 0$, and another with $\mathcal{R}=0$. These solutions will be illustrated in Section III for the case of inflation with any number of interacting scalar fields. The existence of such solutions is well known in special cases, but I do not know of a previous general proof of their existence.

Solutions of this sort are usually called adiabatic, even in contexts where thermodynamics has no relevance. ${ }^{5}$ As we will also see in Section II, in theories in which the energy- momentum tensor is the sum of a number of tensors $T_{f}^{\mu \nu}$ for a set of fluids labeled $f$, we have the stronger result that for $q=0$ in the adiabatic modes, the perturbations in each fluid satisfy

$$
\frac{\delta \rho_{f}}{\dot{\bar{\rho}}_{f}}=\frac{\delta p_{f}}{\dot{\bar{p}}_{f}}=\frac{\delta \rho}{\dot{\bar{\rho}}}=\frac{\delta p}{\dot{\bar{p}}} .
$$

In the special case where the unperturbed energy-momentum tensors are separately conserved, we also have $\dot{\bar{\rho}}_{f}=-3 H\left(\bar{\rho}_{f}+\bar{p}_{f}\right)$, in which case Eq. (4) implies that the ratios $\delta \rho_{f} /\left(\bar{\rho}_{f}+\bar{p}_{f}\right)$ are equal:

$$
\frac{\delta \rho_{f}}{\bar{\rho}_{f}+\bar{p}_{f}}=\frac{\delta \rho}{\bar{\rho}+\bar{p}}
$$

which is often taken to be what is meant by an adiabatic perturbation.

Things appear very different in synchronous gauge. As shown in Section IV, when we take the limit of vanishing wave number in the field equations of synchronous gauge, we find that these equations respect the conservation of a quantity $A$ in all modes whatever the contents of the universe, provided only that none of the perturbations in synchronous gauge blow up in the limit $q \rightarrow$ 0 . At first sight, this presents an apparent paradox. All gauges are equivalent, so how can there be a quantity that is conserved for zero wave number in all modes during all eras in synchronous gauge and no equally universal conservation law for zero wave number in Newtonian gauge? We will find the answer to be that when we speak of the limit $q=0$ we mean different things in different gauges. Though mathematically equivalent, synchronous gauge

\footnotetext{
${ }^{5}$ Sometimes the non-adiabatic solutions of the field equations are called isocurvature perturbations. This is a misnomer, because even for $q=0$ it is only possible for $\mathcal{R}$ to have the constant value zero if $X=0$. Nevertheless, as we will see in Section IV, in synchronous gauge there is a sense in which the solutions that do not correspond to the adiabatic solutions of Newtonian gauge can indeed be regarded as isocurvature modes.
} 
and Newtonian gauge suggest different hypotheses about how perturbations behave in this limit, leading to different conditions for the validity of the conservation law for $q=0$.

In some work on cosmological fluctuations, a quantity $\zeta$, related to the spatial curvature on spacelike surfaces of constant energy density, is used in place of $\mathcal{R}$. It is defined in Newtonian gauge by[5]

$$
\zeta \equiv-\Psi-H \delta \rho / \overline{\bar{\rho}}=-\Psi+\delta \rho / 3(\bar{\rho}+\bar{p}) .
$$

By taking a suitable linear combination of the time-time and time- space components of the Einstein field equation and the part of the space- space component proportional to $\delta_{i j}$, one can derive a general constraint[6],

$$
a^{3} \delta \rho-3 H a^{3}(\bar{\rho}+\bar{p}) \delta u+\left(\frac{q^{2} a}{4 \pi G}\right) \Psi=0,
$$

so that

$$
\mathcal{R}-\zeta=\left(\frac{q^{2}}{3 a^{2} \dot{H}}\right) \Psi
$$

and therefore in all modes $\zeta \rightarrow \mathcal{R}$ in the limit $q \rightarrow 0$.

\section{ADIABATIC MODES IN NEWTONIAN GAUGE}

We consider a general cosmological model, based on the Einstein field equations, supplemented with whatever other equations are needed to give the energy density, pressure, and velocity potential perturbations in terms of independent dynamical variables, and the field equations satisfied by those variables. We will demonstrate the general existence of a pair of adiabatic solutions of these field equations in Newtonian gauge: one with $\mathcal{R} \neq 0$ and constant in the limit $q \rightarrow 0$, and the other with $\mathcal{R}=0$ in this limit. In order to draw these conclusions without specifying the formulas for $\delta \rho, \delta p$, and $\delta u$, we use a trick, based on the fact that although there is no remaining gauge symmetry in Newtonian gauge for $q \neq 0$, for $q=0$ there is a remnant gauge symmetry of the field equations in Newtonian gauge, which makes it easy to find exact general solutions of the field equations for $q=0$. Not all of these solutions are physical. To be physical, such a solution must be the limit as $q \rightarrow 0$ of a solution of the field equations for $q \neq 0$ in at least a neighborhood of $q=0$. For this to be the case, it is necessary for the $q=0$ solution 
to satisfy certain conditions imposed by the Einstein field equations, which limit the physical solutions to a linear combination of just two independent adiabatic modes. We will then make a mild technical assumption about the dependence of the field equations on $q$, which guarantees that these two solutions are the limits as $q \rightarrow 0$ of physical solutions for $q \neq 0$.

Whatever the constituents of the universe, for $q=0$ the field equations in Newtonian gauge for scalar (i.e., compressional) modes will always be invariant under the gauge transformation induced by a redefinition of the time coordinate

$$
t \rightarrow t+\epsilon(t)
$$

and a re-scaling of the space coordinate

$$
x^{i} \rightarrow x^{i}(1-\lambda)
$$

with $\epsilon(t)$ an arbitrary infinitesimal function of time and $\lambda$ an arbitrary infinitesimal constant. For scalar modes, $\delta g_{i 0}$ is proportional to $q_{i}$, and the part of $\delta g_{i j}$ not proportional to $\delta_{i j}$ is proportional to $q_{i} q_{j}$, so both automatically vanish for $q \rightarrow 0$, and we therefore do not need to impose any conditions on $\epsilon(t)$ and $\lambda$ to remain in Newtonian gauge for $q=0$. Eqs. (9) and (10) provide the most general spacetime transformations of purely scalar perturbations that preserve the condition $q=0$.

Under this gauge transformation, the $q=0$ perturbations undergo the transformation

$$
\begin{gathered}
\Psi \rightarrow \Psi+H \epsilon-\lambda, \quad \Phi \rightarrow \Phi-\dot{\epsilon}, \\
\delta \rho \rightarrow \delta \rho-\dot{\bar{\rho}} \epsilon, \quad \delta p \rightarrow \delta p-\dot{\bar{p}} \epsilon .
\end{gathered}
$$

Likewise, in the case of separate fluids with energy density and pressure $\rho_{f}$ and $p_{f}$ or separate scalar fields $\varphi_{f}$,

$$
\delta \rho_{f} \rightarrow \delta \rho_{f}-\dot{\bar{\rho}}_{f} \epsilon, \quad \delta p_{f} \rightarrow \delta p_{f}-\dot{\bar{p}}_{f} \epsilon
$$

or

$$
\delta \varphi_{f} \rightarrow \delta \varphi_{f}-\dot{\bar{\varphi}}_{f} \epsilon
$$

It follows that there is always a solution of the Newtonian gauge field equations for $q=0$, in which:

$$
\Psi=H \epsilon-\lambda, \quad \Phi=-\dot{\epsilon},
$$




$$
\delta \rho=-\dot{\bar{\rho}} \epsilon, \quad \delta p=-\dot{\bar{p}} \epsilon
$$

and for several fluids

$$
\delta \rho_{f}=-\dot{\bar{\rho}}_{f} \epsilon, \quad \delta p_{f}=-\dot{\bar{p}}_{f} \epsilon,
$$

or several scalar fields

$$
\delta \varphi_{f}=-\dot{\bar{\varphi}}_{f} \epsilon,
$$

where $\epsilon(t)$ is an arbitrary function of time and $\lambda$ is an arbitrary constant. (It is not necessary for this that the energy-momentum tensors of the individual fluids or scalar fields be separately conserved; all we need is that they are tensors.)

Of course, for general $\epsilon(t)$ and $\lambda$ this is just a gauge mode. For it to have any physical significance, it must satisfy certain conditions that allow it to be extended to the case of non-zero wave number. In particular, the part of the space-space component of the Einstein field equations that is not proportional to $\delta_{i j}$ takes the form (with $\delta \sigma$ the anisotropic stress):

$$
q_{i} q_{j}(\Phi-\Psi)=-8 \pi G q_{i} q_{j} \delta \sigma
$$

so this equation disappears for $q=0$. In order for the solution (15)-(18) of the field equations to be extended to $q \neq 0$, we must have

$$
\Phi=\Psi-8 \pi G \delta \sigma
$$

and therefore $\epsilon(t)$ and $\lambda$ must satisfy the condition

$$
\dot{\epsilon}+H \epsilon=\lambda-8 \pi G \delta \sigma,
$$

which can always be satisfied by a suitable choice of $\epsilon(t)$.

There is another equation that disappears for $q=0$ : for scalar modes the space-time component of the Einstein field equations reads

$$
q_{i}(-2 \dot{\Psi}-2 H \Phi)=8 \pi G(\bar{\rho}+\bar{p}) q_{i} \delta u=-2 \dot{H} q_{i} \delta u
$$

where $\delta u$ is the velocity potential in Newtonian gauge, which does not appear in the equations for $q=0$. Hence in order for the solution we have found to be extended to $q \neq 0$, this solution must also have a velocity potential given by

$$
\dot{H} \delta u=\dot{\Psi}+H \Phi,
$$


or, using the result (15),

$$
\delta u=\epsilon .
$$

This agrees with what would be found from the gauge transformation induced by the coordinate transformation (9). Likewise, with several fluids,

$$
\delta u_{f}=\epsilon
$$

From Eqs. (16) and (24) it follows that $\delta \rho=3 H(\bar{\rho}+\bar{p}) \delta u$, so the constraint equation (7) is automatically satisfied for $q=0$ by this solution. By inserting Eqs. (15), (16), and (24) in equations (1) and (6), we now find that for $q=0$, this solution has

$$
\mathcal{R}=\zeta=\lambda,
$$

so $\mathcal{R}$ and $\zeta$ are indeed constant and equal for this solution in the limit $q \rightarrow 0$, as was to be shown. They are also non-zero, as long as we take $\lambda \neq 0$.

Now we have to ask what additional conditions are needed to ensure that this solution is the limit as $q \rightarrow 0$ of a solution with $q \neq 0$. In general, any closed set of linear homogeneous ordinary differential equations for a finite set of dependent variables can be put in the first-order form

$$
\dot{y}_{n}(t)+\sum_{m} C_{n m}(t) y_{m}(t)=0 .
$$

(If some of the original set of field equations involve derivatives of higher than first order, we can still write the equations in the form (27) by including some derivatives of the field variables among the $y_{m}(t)$.) This has the general solution

$$
y_{n}(t)=\sum_{m}\left[T\left\{\exp \left(-\int_{t_{0}}^{t} C\left(t^{\prime}\right) d t^{\prime}\right)\right\}\right]_{n m} y_{m}\left(t_{0}\right)
$$

with $t_{0}$ arbitrary, and with $T$ denoting a time-ordered product defined by a power series expansion of the exponential, which for finite matrices is always convergent. The initial conditions may be subject to constraints like Eq. (7), which can be written

$$
\sum_{n} c_{n} y_{n}\left(t_{0}\right)=0
$$

(Eq. (7) is such a constraint, because the equations of energy and momentum conservation and the gravitational field equation $\dot{\Psi}+H \Phi=\dot{H} \delta u$ imply that the left-hand side of Eq. (7) is time-independent.) 
Our "mild technical" assumption is that, as long as the Einstein equations (19) and (22) are written instead in the stronger form (20) and (23), the matrix elements $C_{n m}(t)$ and the constraint coefficients $c_{n}$ are continuous functions of $q$ in at least a neighborhood of $q=0$. In this case the $y_{n}\left(t_{0}\right)$ that satisfy Eq. (29) and the matrix in Eq. (28) will also be continuous in $q$, so that any solution of Eq. (27) for $q=0$ can be extended to a solution for $q \neq 0$ in a neighborhood of $q=0$ by using Eq. (28) and (29) with the values of $C(t)$ and $c$ for $q \neq 0$. The next section shows the validity of this assumption in one illustrative example: inflation with several scalar fields and an arbitrary potential. Because $q$ generally enters the field equations and constraint equations in such a simple manner, we expect that this assumption will always be satisfied, and in any case it is easy to check for any specific model. For $q \neq 0$, there is no remaining gauge freedom in Newtonian gauge (as there is for $q=0$ ), so the adiabatic solution found in this way will be the limit as $q \rightarrow 0$ of a physical solution, not a mere gauge mode.

We expect the anisotropic stress coefficient $\delta \sigma$ for a wide class of theories to be some linear combination of $\delta u, \delta \rho$, and $\delta p$, so that for the solution (15)-(17), (24)-(25), $\delta \sigma$ may be written as $\delta \sigma=\epsilon \Sigma$, with $\Sigma(t)$ depending only on unperturbed quantities. (For instance, a non-zero shear viscosity $\eta$ in an imperfect fluid gives $\delta \sigma=-2 \eta \delta u$, so here $\Sigma=-2 \eta$.) In all such cases, Eq. (21) has the general solution

$$
\epsilon(t)=\frac{\lambda}{\alpha(t)} \int^{t} \alpha\left(t^{\prime}\right) d t^{\prime}
$$

where

$$
\alpha(t) \equiv a(t) \exp \left(8 \pi G \int^{t} \Sigma\left(t^{\prime}\right) d t^{\prime}\right)
$$

and the lower limit on the integral in Eq. (30) is arbitrary.

There is also a second mode, corresponding to the possibility of shifting the lower limit of the integral in Eq. (30), for which $\epsilon(t)$ goes as $\epsilon(t) \propto 1 / \alpha(t)$. Since shifting the lower bound on the integral in Eq. (30) has no effect on the value (26) of $\mathcal{R}$ and $\zeta$, this solution has $\mathcal{R}=\zeta=0$.

In the special case of vanishing anisotropic stress we have $\delta \sigma=0$, so here $\Phi=\Psi$, and $\alpha(t)$ is just the Robertson-Walker scale factor $a(t)$. The general solution of Eq. (21) is then

$$
\epsilon(t)=\frac{\lambda}{a(t)} \int^{t} a\left(t^{\prime}\right) d t^{\prime}
$$


with an arbitrary lower limit. This eventually increases in absolute value as $t$ for Robertson-Walker scale factors that grow as any power of $t$, while in the other mode $\epsilon(t) \propto 1 / a(t)$ decreases with time. Inserting the result (32) in Eqs. (15)-(18) gives explicit results for the perturbations in the gravitational field and various pressures and energy densities as functions of time.

The results presented in this section can be interpreted in terms of what Liddle and Lyth in ref. [1] call a "separate universe" picture, which in one form or another has been used since the beginning of inflationary theory to deal with cosmological fluctuations in the case of a single scalar field. For instance, Bardeen, Steinhardt, and Turner in ref. [5] gave what they called a 'heuristic argument' that in this case any portion of the universe that is larger than the horizon $1 / H$ but smaller than the physical perturbation wavelength $a / q$ would have to look like a separate unperturbed universe, with $\varphi+\delta \varphi$ following the unique evolutionary path of the scalar field, and with all of these separate universes therefore the same except for a variation in the time at which the scalar field satisfies some specific initial condition a few Hubble times after horizon exit. As pointed out by Bardeen et al., it follows then that $\delta \rho / \overline{\bar{\rho}}=\delta p / \dot{\bar{p}}$, and hence $X=0$, for $q / a \ll H$.

There is a potential problem with this sort of argument, that there are two fields involved, the inflaton and the gravitational field, so that different separate universes might have different ratios of these fields. The argument of Bardeen et al. was formulated in a gauge in which it is unnecessary to consider fluctuations in the gravitational field, but it applies also to Newtonian gauge, because in this gauge the constraint (7) allows the gravitational potential to be expressed in terms of fluctuations in the scalar field. But as we have seen in this section, in Newtonian gauge it is necessary not only to allow shifts in the time at which the scalar field reaches some given value after horizon crossing, but also to re-scale the co-moving coordinates used in each separate universe. In synchronous gauge there is no constraint like Eq. (7) that allows us to express the gravitational field in terms of the scalar field, and so, as we will see in Section IV, the solutions even for inflation with a single scalar field do not satisfy $X=0$ in the limit $q \rightarrow 0$.

There is another potential problem, that the equation of motion of the scalar field is a second-order differential equation, so that there are two independent solutions whose relative coefficients may vary from one separate universe to another. Bardeen et al. and other authors avoid this problem by assuming that the scalar field experiences a period of "slow roll" inflation, 
in which the differential equation satisfied by the scalar field is of first order, to a good approximation. We have not had to make this assumption, for a reason already pointed out by Guth and $\mathrm{Pi}[7]$ : the Wronskian of these two solutions decays rapidly after horizon crossing, so that it is as if there were only one independent solution. (Guth and Pi considered the case of $H$ constant, but even with a time-dependent $H$ the Wronskian still decays, though not precisely exponentially.)

In any case, it has always been clear that such "separate universe" arguments do not rule out non-adiabatic solutions in the case of several scalar fields, where ratios of the scalar fields may vary from one "separate universe" to another. The results of this section may be interpreted as the statement that in this and all other cases it is always possible to find an adiabatic solution of the field equations in Newtonian gauge in which the separate universes appear the same, except for a shift in the time coordinate and a re-scaling of the co-moving space coordinates.

\section{AN EXAMPLE: MULTIFIELD INFLATION}

For illustration, and to confirm the reasoning of the theorem of the previous section in a case where $X$ does not vanish for all modes, let us consider the case of inflation with an arbitrary number of scalar fields $\varphi_{f}$, and with a general potential $V$ that may include interactions among the various scalars. The energy-momentum tensor of the scalar fields has the perfect-fluid form, so here $\sigma=0$, and $\Phi=\Psi$. The field equations in Newtonian gauge are

$$
\begin{gathered}
\dot{\Psi}+H \Psi=4 \pi G \sum_{f} \dot{\bar{\varphi}}_{f} \delta \varphi_{f} \\
\delta \ddot{\varphi}_{f}+3 H \delta \dot{\varphi}_{f}+\sum_{f^{\prime}} \frac{\partial^{2} V(\bar{\varphi})}{\partial \bar{\varphi}_{f} \partial \bar{\varphi}_{f^{\prime}}} \delta \varphi_{f^{\prime}}+\left(\frac{q^{2}}{a^{2}}\right) \delta \varphi_{f}=-2 \Psi \frac{\partial V(\bar{\varphi})}{\partial \bar{\varphi}_{f}}+4 \dot{\Psi}_{\bar{\varphi}_{f}},
\end{gathered}
$$

and the constraint (7) is here

$$
\left(\dot{H}+\frac{q^{2}}{a^{2}}\right) \Psi=4 \pi G \sum_{f}\left(-\dot{\bar{\varphi}}_{f} \delta \dot{\varphi}_{f}+\ddot{\bar{\varphi}}_{f} \delta \varphi_{f}\right) .
$$

We can write Eqs. (33) and (34) in the form (27) by taking the $y_{n}$ to run over $\Psi$ and all $\phi_{f}$ and $\dot{\phi}_{f}$, in which case the constraint (35) is of the form 
(29). Here obviously $C_{n m}(t)$ and $c_{n}$ are continuous in $q$ in a neighborhood of $q=0$; in fact, they are just linear functions of $q^{2}$. Hence any solution of Eqs. (33)-(35) that we find for $q=0$ can be extended to a solution for $q \neq 0$.

Let us try for a solution for $q=0$ in which all of the individual velocity potentials $-\delta \varphi_{f} / \dot{\bar{\varphi}}_{f}$ are equal, so that

$$
\delta \varphi_{f}=-\dot{\bar{\varphi}}_{f} \delta u
$$

with the common value satisfying

$$
\delta \dot{u}=-\Psi
$$

Using the time-derivative of the unperturbed scalar field equation

$$
\ddot{\bar{\varphi}}_{f}+3 H \dot{\bar{\varphi}}_{f}+\frac{\partial V(\bar{\varphi})}{\partial \bar{\varphi}_{f}}=0
$$

we can put Eq. (34) for $q=0$ in the form

$$
\dot{H} \delta u+H \delta \dot{u}+\delta \ddot{u}=0 .
$$

Also, the gravitational field equation (33) now reads $\dot{\Psi}+H \Psi=\dot{H} \delta u$, which Eq. (39) guarantees is automatically satisfied by the $\Psi$ given by Eq. (37). The general solution is

$$
\delta u=\frac{\lambda}{a} \int a d t, \quad \Psi=H \delta u-\lambda
$$

(with $\lambda$ an arbitrary constant), just as we found above in Eqs. (15), (24), and (32). The perturbations to the energy density and pressure of the $f$ th field here are

$$
\delta \rho_{f}=-\Psi \dot{\bar{\varphi}}^{2}+\dot{\bar{\varphi}} \delta \dot{\varphi}+\frac{\partial V}{\partial \bar{\varphi}_{f}} \delta \varphi_{f}=-(\Psi+\delta \dot{u}) \dot{\bar{\varphi}}_{f}^{2}-\dot{\bar{\rho}}_{f} \delta u=-\dot{\bar{\rho}}_{f} \delta u
$$

and

$$
\delta p_{f}=-\Psi \dot{\bar{\varphi}}^{2}+\dot{\bar{\varphi}} \delta \dot{\varphi}-\frac{\partial V}{\partial \bar{\varphi}_{f}} \delta \varphi_{f}=-(\Psi+\delta \dot{u}) \dot{\bar{\varphi}}_{f}^{2}-\dot{\bar{p}}_{f} \delta u=-\dot{\bar{p}}_{f} \delta u
$$

so this mode is adiabatic, in the sense that $X \rightarrow 0$ for $q \rightarrow 0$. Inserting Eq. (40) in Eq. (1) gives again $\mathcal{R}=\lambda$. 
Once again, because of the freedom to shift the lower limit of the integral in Eq. (40), there are two adiabatic modes here, the second with $\delta u \propto 1 / a$ and $\mathcal{R}=0$.

For a single scalar field, Eqs. (33) and (34) are a third-order set of differential equations, and therefore have a third independent solution. The third solution can also be found explicitly, and turns out to have $\dot{\mathcal{R}} \propto 1 / a^{3} \dot{H}$ for $q=0$, so this solution is not adiabatic. However, this third solution is eliminated by the constraint Eq. (35), which as we have seen in the previous section is automatically satisfied by any adiabatic solution, but is not satisfied by the non-adiabatic solution of Eqs. (33) and (34). For $N$ scalar fields Eqs. (33) and (34) have $2 N+1$ independent solutions, of which two are adiabatic, and one is eliminated by Eq. (35), leaving $2 N-2$ non-adiabatic solutions.

\section{SYNCHRONOUS GAUGE}

We now turn to synchronous gauge. With zero unperturbed spatial curvature, the perturbed metric has components

$$
g_{i j}(\mathbf{x}, t)=a^{2}(t) \delta_{i j}+h_{i j}(\mathbf{x}, t), \quad g_{00}=-1, \quad g_{i 0}=0,
$$

with $h_{i j}$ a small perturbation. We now assume for simplicity that the perturbed energy-momentum tensor takes the perfect-fluid form

$$
T_{\mu \nu}=p g_{\mu \nu}+(p+\rho) u_{\mu} u_{\nu} .
$$

The unperturbed quantities $\bar{p}$ and $\bar{\rho}$ depend only on time, and the unperturbed velocity four-vector has components $\bar{u}^{0}=1, \bar{u}^{i}=0$. The normalization condition $u_{\mu} u^{\mu}=-1$ then requires that the velocity perturbation $\delta u_{\mu}^{(S)}$ is purely spatial. (A superscript $(S)$ is used to denote perturbed quantities in synchronous gauge.) We consider only compressional modes, for which $\delta u_{i}^{(S)}=\partial \delta u^{(S)} / \partial x^{i}$. Then the relevant field equations for a Fourier component with wave number $q$ are[8]

$$
\frac{d}{d t}\left(a^{2} \psi\right)=-4 \pi G a^{2}\left(\delta \rho^{(S)}+3 \delta p^{(S)}\right)
$$

and

$$
\delta \dot{\rho}^{(S)}+3 H\left(\delta \rho^{(S)}+\delta p^{(S)}\right)=-(\bar{\rho}+\bar{p})\left(\psi-a^{-2} q^{2} \delta u^{(S)}\right)
$$


Here $\psi$ is a field employed in recent work using synchronous gauge[9]

$$
\psi \equiv \frac{d}{d t}\left(\frac{h_{i i}}{2 a^{2}}\right)
$$

There is also an Euler equation that will be needed later in this section:

$$
\frac{d}{d t}\left[a^{3}(\bar{\rho}+\bar{p}) \delta u^{(S)}\right]=-a^{3} \delta p^{(S)} .
$$

From equations (45) and (46) together with the relation $4 \pi G(\bar{\rho}+\bar{p})=-\dot{H}$ it follows that

$$
\dot{A}=-q^{2} H \delta \dot{u}^{(S)}
$$

where

$$
A \equiv a^{2} H \psi-4 \pi G a^{2} \delta \rho^{(S)}-q^{2} H \delta u^{(S)} .
$$

Here is the proof: Eq. (46) can be written

$$
\delta \dot{\rho}^{(S)}+3 H\left(\delta \rho^{(S)}+\delta p^{(S)}\right)=\frac{\dot{H}}{4 \pi G}\left(\psi-a^{-2} q^{2} \delta u^{(S)}\right)
$$

and it follows immediately from Eq. (45) that

$$
\frac{d\left(a^{2} H \psi\right)}{d t}=-4 \pi G a^{2} H\left(\delta \rho^{(S)}+3 \delta p^{(S)}\right)+a^{2} \dot{H} \psi
$$

Eliminating $\dot{H} \psi$ from these two equations gives

$\frac{d\left(a^{2} H \psi\right)}{d t}=-4 \pi G a^{2} H\left(\delta \rho^{(S)}+3 \delta p^{(S)}\right)+4 \pi G a^{2}\left[\delta \dot{\rho}^{(S)}+3 H\left(\delta \rho^{(S)}+\delta p^{(S)}\right)\right]+q^{2} \dot{H} \delta u^{(S)}$

or in other words

$$
\frac{d}{d t}\left[a^{2} H \psi-4 \pi G a^{2} \delta \rho^{(S)}\right]=q^{2} \dot{H} \delta u^{(S)}
$$

The quantity in square brackets on the left is not invariant under the gauge transformations that preserve the condition (43) for synchronous gauge, so instead we work with the related gauge-invariant quantity (50), for which Eq. (49) follows immediately. 
As long as the velocity potential remains finite in the limit $q \rightarrow 0$, Eq. (49) yields a conservation law

$$
\dot{A}=0 \text { for } q=0 \text {. }
$$

This is true for all modes in all cases, including inflation with several scalar fields and for the transition from radiation to matter dominance. The conservation of $A$ in the limit $q=0$ can also be derived by simply perturbing $a(t), \rho(t)$, and the curvature constant $K$ in the Friedmann equation, which gives $\delta K=-2 A / 3$.

By taking suitable linear combinations of solutions, it is always possible to arrange that for $q=0$ just one of a complete set has $A \neq 0$, while all the other solutions have $A=0$. Examples are given in an appendix to this paper. Because of the connection of $A$ with the spatial curvature, it is legitimate to call the solutions with $A=0$ isocurvature modes. When $q$ is small but non-zero the isocurvature solutions usually have both $A$ and $\dot{A}$ of order $q^{2}$, so that Eq. (49) does not keep $A$ for these solutions from undergoing large fractional changes. This does not vitiate the usefulness of the conservation law for initial conditions that give a physical perturbation in which all solutions make contributions with comparable coefficients. In this case, the contribution of the isocurvature modes to $A$ may be rapidly varying, but at any given time they will be small as long as $q$ is sufficiently small. The physical solution will have a rapid fractional variation in $A$ only if the coefficient of the mode with $A \neq 0$ for $q=0$ is suppressed, or if the coefficients of the isocurvature modes are enhanced.

There is a simple relation between the quantity $A$ introduced in this section and the more familiar quantity $\mathcal{R}$ discussed in Sections I-III. Given perturbations $\Psi, \delta \rho$ and $\delta u$ in Newtonian gauge, we can find the perturbations $\psi, \delta \rho^{(S)}$, and $\delta u^{(S)}$ in synchronous gauge from the transformation equations:

$$
\psi=-3 \dot{\Psi}-3 \frac{d}{d t}(H \epsilon)+(q / a)^{2} \epsilon, \quad \delta \rho^{(S)}=\delta \rho-\epsilon \dot{\bar{\rho}} \quad \delta u^{(S)}=\delta u+\epsilon,
$$

where

$$
\dot{\epsilon}=\Psi \text {. }
$$

(The possibility of shifting $\epsilon$ by a constant term corresponds to the possibility of making gauge transformations that preserve the conditions for synchronous 
gauge.) By applying these equations to the quantity (50), it is elementary to show that $A$ is related to the quantity $\mathcal{R}$ defined in Eq. (1) by

$$
A=-q^{2} \mathcal{R} .
$$

Thus for any finite $q$ the fractional rate of change in $\mathcal{R}$ will be the same as the fractional rate of change in $\mathcal{A}$. In some treatments of multi-field inflation[10] and in discussions of the curvaton model[4], it is simply assumed that the mode with $\mathcal{R} \neq 0$ and hence $A \neq 0$ is somehow suppressed, which is enough to explain why these authors find a significant fractional change in $\mathcal{R}$. But why more generally does the condition $X=0$ play an important role in establishing the conservation of $\mathcal{R}$ for $q \rightarrow 0$ in Newtonian gauge, while there seems to be no similar condition needed for the conservation of $A$ in synchronous gauge?

As a first step toward resolving this apparent paradox, we note from Eq. (54) that that the limit as $q \rightarrow 0$ of the perturbed quantities $\delta \rho^{(S)}$ and $\psi$ in synchronous gauge in the mode for which $A \neq 0$ in this limit is not obtained by applying a gauge transformation to the perturbed quantities in the corresponding mode in Newtonian gauge for $q=0$, since that would give $A=0$ for $q=0$. We can go further, and show in general that for $q=0$, the synchronous gauge solution corresponding to any adiabatic solution of the Newtonian gauge field equations (normalized to not diverge as $q \rightarrow 0$ ) has vanishing values not only for $A$, but also (up to a choice of a particular synchronous gauge) for $\psi$ and the total density fluctuation $\delta \rho^{(S)}$ and velocity potential $\delta u^{(S)}$.

The reasoning here is essentially the reverse of that used to prove the theorem of Section II. We will use the space-time component of the Einstein field equations in Newtonian gauge

$$
\dot{\Psi}+H \Psi=-4 \pi G(\bar{\rho}+\bar{p}) \delta u=\dot{H} \delta u .
$$

We work in the limit $q=0$, assuming that the solution is normalized so that in Newtonian gauge all fluctuations remain finite in this limit. (As we shall see, this assumption is less innocent than it may seem.) Then for modes that for $q=0$ are adiabatic in the sense that $X=0$, Eqs. (1) and (2) give

$$
\dot{\Psi}=\frac{d}{d t}(H \delta u)
$$


for $q=0$. Combining this with Eq. (55) gives

$$
\Psi=-\delta \dot{u} .
$$

Thus according to Eq. (53) we can adopt a particular synchronous gauge such that the transformation parameter $\epsilon$ in Eq. (52) is

$$
\epsilon=-\delta u
$$

Using Eqs. (56) and (58) in Eq. (52) shows immediately that, for $q=0$,

$$
\psi=0 \text {. }
$$

Furthermore, Eq. (55) together with the Newtonian gauge Euler equation supplies a general constraint equivalent to Eq. (7) for $q=0$ :

$$
-4 \pi G \delta \rho=3 H \dot{H} \delta u
$$

Eqs. (52) and (60) give the synchronous gauge density fluctuation

$$
\begin{aligned}
-4 \pi G \delta \rho^{(S)} & =-4 \pi G[\delta \rho-\epsilon \dot{\bar{\rho}}] \\
& =3 H \dot{H} \delta u+[-4 \pi G] \delta u[-3 H(\bar{\rho}+\bar{p})] \\
& =0
\end{aligned}
$$

Finally, the velocity potential in this synchronous gauge is

$$
\delta u^{(S)}=\delta u+\epsilon=0 .
$$

Thus no synchronous gauge perturbation with non-vanishing values of $\psi$ or $\delta \rho^{(S)}$ or $\delta u^{(S)}$ (apart from those that can be eliminated by a transformation to a different synchronous gauge), such as modes 1,2 , and 3 of the radiation plus cold dark matter model of the appendix, can be the gauge transformation of one of the $q=0$ adiabatic Newtonian gauge solutions. Rather, the synchronous gauge solutions for $q=0$ with $A$ a non-zero constant must be the gauge transformations of the terms of order $q^{2}$ in the adiabatic Newtonian gauge solution with $\mathcal{R} \neq 0$, re-normalized by dividing by a factor $q^{2}{ }^{6}$ With

${ }^{6}$ This is why it is possible for the quantity $X$ not to vanish in any mode for $q=0$ in synchronous gauge, as we find in the appendix in the case of inflation, while there are two modes in Newtonian gauge in which $X \rightarrow 0$ for $q \rightarrow 0$, despite the fact that $X$ is gauge invariant. It is not that $X$ is different in the two gauges, but rather that the limit $q \rightarrow 0$ means different things in synchronous and Newtonian gauge. 
this re-normalization of the synchronous gauge modes, as in the appendix, the conserved quantity $A$ is not necessarily of order $q^{2}$, as would be expected from Eq. (54), but can have a finite limit for $q \rightarrow 0$, as we will find it does in the appendix.

Now at last we come to the point. Working in Newtonian gauge, it is most natural to assume that, with an over-all normalization factor chosen so that $\mathcal{R}$ is finite and non-zero in the limit $q=0$, all density fluctuations and velocity potentials as well as $\Psi$ are non-zero in this limit. Under this assumption, if the contribution of non-adiabatic modes is comparable to that of the adiabatic modes, $\mathcal{R}$ will undergo significant changes with time. Transforming this sort of solution to synchronous gauge, we have found above that the density fluctuations and the total velocity potential receive contributions of order $q^{2}$ (relative to the Newtonian gauge perturbations) from the adiabatic modes but of order unity from the non-adiabatic modes, so that $\dot{A}$ is of order $q^{2}$, while $A$ is also of order $q^{2}$, and so $A$ does suffer significant changes with time. Or we can re-normalize the synchronous gauge fluctuations by an over-all factor of order $1 / q^{2}$, in which case $A$ and the density fluctuations and velocity potentials receive contributions of order unity for $q=0$ from the adiabatic modes, as in the appendix, while the contribution of the non-adiabatic modes to the total velocity potential if present is enhanced by a peculiar looking factor of $1 / q^{2}$, giving both $A$ and $\dot{A}$ non-zero limits for $q \rightarrow 0$.

On the other hand, working in synchronous gauge, it is most natural to assume that, with an over-all normalization factor chosen so that $A$ is finite and non-zero in the limit $q=0$, all density fluctuations and velocity potentials as well as $\psi$ are finite in this limit. Under this assumption, it makes no difference whether the contribution of non-adiabatic modes is comparable to that of the adiabatic modes; even if it is, $A$ will undergo no significant changes with time. Transforming this sort of solution to Newtonian gauge, one finds that the density fluctuations and the total velocity potential receive contributions of order $1 / q^{2}$ from the adiabatic modes and of order unity from the non-adiabatic modes, so $\mathcal{R}$ is of order $1 / q^{2}$ while its rate of change is only of order unity. Or we can re- normalize the Newtonian gauge fluctuations by an over-all factor of order $q^{2}$, in which case $\mathcal{R}$ and the density fluctuations and velocity potentials receive contributions of order unity for $q=0$, while the contribution of any non-adiabatic modes is suppressed by a peculiar looking factor of $q^{2}$, giving $\mathcal{R}$ a zero rate of change for $q=0$. 
So which is right? The issue is not the over-all normalization of the total perturbations, but the relative magnitude of its adiabatic and non-adiabatic terms in the limit $q \rightarrow 0$. There is nothing about either gauge that makes it a more reliable guide to our intuition about this than the other.

It is generally expected that inflation with several scalar fields the general solution does not have $\mathcal{R}$ approaching a constant for increasing $a(t)$, in agreement with what would be expected from the behavior for $q \rightarrow 0$ suggested by Newtonian gauge but not synchronous gauge. But there are cases of multi-field inflation in which $A$ and hence $\mathcal{R}$ do approach constants as $a(t)$ increases, as would be expected from the behavior for $q \rightarrow 0$ suggested by synchronous gauge but not Newtonian gauge. One case is a potential given by a sum of exponentials[11].

$$
V=\sum_{n} g_{n} \exp \left(-\lambda_{n} \varphi_{n}\right)
$$

Another is a potential of the form

$$
V=F\left(\sum_{n} \varphi_{n}^{2}\right),
$$

with $F$ an arbitrary function. It would be interesting to characterize the general class of potentials for multi-field inflation for which $A$ and $\mathcal{R}$ approach constants as $a(t)$ increases.

\section{APPENDIX: LONG-WAVELENGTH SOLUTIONS IN SYNCHRONOUS GAUGE}

In this appendix we will study several examples of calculations for zero wave number in synchronous gauge, to exhibit both solutions with $A \neq 0$ and those with $A=0$. All quantities here will be in synchronous gauge, so we will drop the label $(S)$.

As a first example, consider inflation with just a single real scalar field $\varphi=\bar{\varphi}(t)+\delta \varphi(\mathbf{x}, t)$, and potential $V(\varphi)$. As is well known, the unperturbed pressure and energy density are

$$
\bar{\rho}=\frac{1}{2} \dot{\bar{\varphi}}^{2}+V(\bar{\varphi}), \quad \bar{p}=\frac{1}{2} \dot{\bar{\varphi}}^{2}-V(\bar{\varphi}),
$$


from which we find the equation of motion of the unperturbed scalar field

$$
\ddot{\bar{\varphi}}+3 H \dot{\bar{\varphi}}+V^{\prime}(\bar{\varphi})=0
$$

The perturbations to the energy density and pressure are

$$
\delta \rho=\dot{\bar{\varphi}} \delta \dot{\varphi}+V^{\prime}(\bar{\varphi}) \delta \varphi, \quad \delta p=\dot{\bar{\varphi}} \delta \dot{\varphi}-V^{\prime}(\bar{\varphi}) \delta \varphi .
$$

Also, the perturbed velocity potential is

$$
\delta u=-\delta \varphi / \dot{\bar{\varphi}}
$$

The field equations (45) and (46) for the Fourier component of the perturbations with wave number $q$ here take the form

$$
\begin{gathered}
\frac{d}{d t}\left(a^{2} \psi\right)=-4 \pi G a^{2}\left(4 \dot{\bar{\varphi}} \delta \dot{\varphi}-2 V^{\prime}(\bar{\varphi}) \delta \varphi\right), \\
\delta \ddot{\varphi}+3 H \delta \dot{\varphi}+V^{\prime \prime}(\bar{\varphi}) \delta \varphi+a^{-2} q^{2} \delta \varphi=-\dot{\bar{\varphi}} \psi,
\end{gathered}
$$

where

$$
H \equiv \frac{\dot{a}}{a}=\sqrt{\frac{8 \pi G}{3}\left(\frac{\dot{\varphi}^{2}}{2}+V(\bar{\varphi})\right)} .
$$

The Euler equation (48) gives no new information here.

There is a gauge mode, with $\varphi=\tau \dot{\bar{\varphi}}$ and $\psi=\tau\left(3 \dot{H}-q^{2} / a^{2}\right)$, where $\tau$ is an arbitrary time-independent function of $q$. Knowing this solution allows us to reduce the degree of equations (67) and (68) from three to two, in agreement with the number of physical solutions found in Newtonian gauge in Section III. We introduce time-dependent functions $f$ and $g$ by writing

$$
\delta \varphi=f \dot{\bar{\varphi}}, \quad \psi=(f+g)\left(3 \dot{H}-q^{2} / a^{2}\right) .
$$

Equations (67) and (68) then become a second-order set of equations for the gauge-invariant quantities $\dot{f}$ and $g$ :

$$
\begin{gathered}
\ddot{f}+3 H \dot{f}+(\ddot{H} / \dot{H}) \dot{f}=-\left(3 \dot{H}-q^{2} / a^{2}\right) g, \\
\left(3 \dot{H}-q^{2} / a^{2}\right) \dot{g}+(6 H \dot{H}+3 \ddot{H}) g=\left(\dot{H}+q^{2} / a^{2}\right) \dot{f}
\end{gathered}
$$


in which the gauge mode appears in the possibility of adding a constant to $f$. These equations can be solved exactly for $q=0$ and $H(t)$ arbitrary. There are two physical solutions:

\section{Mode 1:}

$g^{(1)}(t)=\frac{1}{3 a^{3}(t) \dot{H}(t)} \int_{0}^{t} a\left(t^{\prime}\right) d t^{\prime}, \quad \dot{f}^{(1)}(t)=\frac{1}{a^{2}(t) \dot{H}(t)}\left[1-\frac{H(t)}{a(t)} \int_{0}^{t} a\left(t^{\prime}\right) d t^{\prime}\right]$.

Mode 2:

$$
g^{(2)}(t)=\frac{1}{3 a^{3}(t) \dot{H}(t)}, \quad \dot{f}^{(2)}(t)=-\frac{H(t)}{a^{3}(t) \dot{H}(t)}
$$

(The lower limit 0 on the integral over $t^{\prime}$ is arbitrary; changing it just amounts to adding some of mode 2 to mode 1.)

Equation (50) gives the values of $A$ for $q=0$ in these two modes as the constants

$$
A_{1}=1, \quad A_{2}=0,
$$

even though neither of these solutions satisfies the adiabatic condition $X=0$. A general mixture of modes with coefficients $c_{1}$ and $c_{2}$ will have $A=c_{1}$ for $q=0$, provided $c_{2}$ does not blow up in this limit. With this proviso, the conservation of $A$ allows the value of $c_{1}$ that is calculated for a given inflaton potential to be used to find the strength of the non-isocurvature mode at later times. However, if the value of $c_{2}$ for the physical solution found after horizon crossing went as $c / q^{2}$ for $q \rightarrow 0$, while $c_{1}$ remained finite, then Eqs. (49), (66), (70), and (74) would give

$$
\dot{A} \rightarrow c_{2} \dot{A}_{2} \rightarrow \frac{-c H^{2}(t)}{a^{3}(t) \dot{H}(t)} \quad \text { for } \quad q \rightarrow 0
$$

and there would be no useful conservation law even for $q=0$. As discussed in Section IV, this is just what we would expect if we assumed that, with an over-all normalization factor chosen so that $\mathcal{R}$ is finite and non-zero in the limit $q=0$, all density fluctuations and velocity potentials in Newtonian gauge as well as $\Psi$ are finite and non-zero in this limit.

For another example, we consider a later epoch, when the dominant constituents of the universe were radiation and cold dark matter. (For simplicity, 
we are neglecting the baryon density compared with the density of cold dark matter, but supposing that there are still enough baryons to keep the radiation in thermal equilibrium, and we are ignoring the effects of free-streaming neutrinos.) We adopt a particular synchronous gauge in which the cold dark matter is at rest. The field equations then are Eqs. (46) and (48) for the radiation energy density perturbation $\delta \rho_{R}$ and velocity potential $\delta u_{R}$; Eq. (46) for the cold dark matter density $\rho_{D}$; and Eq. (45) for $\psi$, with the total energy density and pressure appearing on the right hand side:

$$
\begin{gathered}
\delta \dot{\rho}_{R}+4 H \delta \rho_{R}=-(4 / 3) \bar{\rho}_{R}\left(\psi-a^{-2} q^{2} \delta u_{R}\right), \\
4 \frac{d}{d t}\left[a^{3} \bar{\rho}_{R} \delta u_{R}\right]=-a^{3} \delta \rho_{R}, \\
\delta \dot{\rho}_{D}+3 H \delta \rho_{D}=-\bar{\rho}_{D} \psi \\
\frac{d}{d t}\left(a^{2} \psi\right)=-4 \pi G a^{2}\left(2 \delta \rho_{R}+\delta \rho_{D}\right) .
\end{gathered}
$$

The unperturbed radiation and dark matter densities go as $a^{-4}$ and $a^{-3}$, respectively. It is convenient here to normalize $a$ so that $a=1$ when $\bar{\rho}_{R}=\bar{\rho}_{D}$, so that

$$
\bar{\rho}_{R}=\rho_{E Q} a^{-4}, \quad \bar{\rho}_{D}=\rho_{E Q} a^{-3},
$$

where $\rho_{E Q}$ is constant.

Equations (78)-(81) are a fourth-order system of differential equations, so there are four modes, all of which are physical because the gauge has been fixed by choosing $\delta u_{D}=0$. For $q=0$, they take the form

\section{Mode 1:}

$$
\begin{aligned}
\delta \rho_{R}^{(1)} & =\frac{1}{\pi G a^{6}}\left(16+8 a-2 a^{2}+a^{3}\right), \\
\delta u_{R}^{(1)} & =-\frac{a}{4 \rho_{E Q}} \sqrt{\frac{3}{8 \pi G \rho_{E Q}}} \int^{a} \frac{a^{4} d a}{\sqrt{1+a}} \delta \rho_{R}^{(1)}, \\
\delta \rho_{D}^{(1)} & =\frac{3}{4 \pi G a^{5}}\left(16+8 a-2 a^{2}+a^{3}\right), \\
\psi^{(1)} & =2 \sqrt{\frac{3}{8 \pi G \rho_{E Q}}} \frac{\sqrt{1+a}}{a^{4}}\left(32+8 a-a^{3}\right)
\end{aligned}
$$


Mode 2:

$$
\begin{aligned}
\delta \rho_{R}^{(2)} & =\frac{1}{\pi G a^{6}} \sqrt{1+a} \\
\delta u_{R}^{(2)} & =-\frac{a}{4 \rho_{E Q}} \sqrt{\frac{3}{8 \pi G \rho_{E Q}}} \int^{a} \frac{a^{4} d a}{\sqrt{1+a}} \delta \rho_{R}^{(2)}, \\
\delta \rho_{D}^{(2)} & =\frac{3}{4 \pi G a^{5}} \sqrt{1+a} \\
\psi^{(2)} & =\sqrt{\frac{3}{8 \pi G \rho_{E Q}}}(4+3 a)
\end{aligned}
$$

Mode 3:

$$
\begin{aligned}
& \delta \rho_{R}^{(3)}=\delta \rho_{D}^{(3)}=\psi^{(3)}=0 \\
& \delta u_{R}^{(3)} \propto a
\end{aligned}
$$

Mode 4:

$$
\begin{aligned}
\delta \rho_{R}^{(4)} & =\frac{1}{\pi G a^{6}}\left(8+4 a-a^{2}-8 \sqrt{1+a}\right), \\
\delta u_{R}^{(4)} & =-\frac{a}{4 \rho_{E Q}} \sqrt{\frac{3}{8 \pi G \rho_{E Q}}} \int^{a} \frac{a^{4} d a}{\sqrt{1+a}} \delta \rho_{R}^{(3)}, \\
\delta \rho_{D}^{(4)} & =\frac{3}{8 \pi G a^{5}}(8+4 a-8 \sqrt{1+a}), \\
\psi^{(4)} & =\frac{8}{a^{4}} \sqrt{\frac{3}{8 \pi G \rho_{E Q}}}((4+a) \sqrt{1+a}-4-3 a)
\end{aligned}
$$

The lower bound on the integrals in the formulas for $\delta u_{R}$ in modes 1,2 , and 4 are arbitrary; changing this lower limit in any of these integrals just amounts to adding some of mode 3 to that mode.

Note that modes 1,2 , and (trivially) 3 are adiabatic, in the sense that

$$
\frac{\delta \rho_{D}}{\bar{\rho}_{D}}=\frac{\delta \rho_{R}}{\bar{\rho}_{R}+\bar{p}_{R}},
$$

(and so $X=0$ ) while mode 4 is not adiabatic in this sense. The values of $A$ for the four modes are

$$
A_{1}=1, \quad A_{2}=A_{3}=A_{4}=0 .
$$


Thus modes 2 and 3 are both adiabatic and isocurvature. An arbitrary mixture of modes will have $A$ constant unless the coefficients of modes 2,3 , or 4 blow up as $1 / q^{2}$ limit $q \rightarrow 0$, which will be the case if the fluctuations in the non-adiabatic modes in Newtonian gauge have non-zero limits for $q \rightarrow 0$.

\section{ACKNOWLEDGMENTS}

I am grateful for helpful correspondence with E. Bertschinger, D. Lyth, S. Mukhanov, and N. Turok. This research was supported in part by the Robert A. Welch Foundation and NSF Grants PHY-0071512 and PHY-9511632.

\section{REFERENCES}

1. J. M. Bardeen, Phys. Rev. D22, 1882 (1980); D. H. Lyth, Phys. Rev. D31, 1792 (1985). For reviews, see J. Bardeen, in Cosmology and Particle Physics, eds. Li-zhi Fang and A. Zee (Gordon \& Breach, New York, 1988); A. R. Liddle and D. H. Lyth, Cosmological Inflation and Large Scale Structure (Cambridge University Press, Cambridge, UK, 2000).

2. J. M. Bardeen, Phys. Rev. D22, 1882 (1980), Eq. (5.21).

3. E. Bertschinger, in Cosmology and Large Scale Structure - Proceedings Session LX of the Les Houches Summer School, ed. R. Schaeffer, J. Silk, M. Spiro, and J. Zinn-Justin (Amsterdam: Elsevier Science, 1996).

4. S. Mollerach, Phys. Rev. D 42, 313 (1990); A. D. Linde and V. Mukhanov, Phys. Rev. D 56, 535 (1997); D. H. Lyth and D. Wands, Phys. Lett. B 524, 5 (2002); T. Moroi and T. Takahashi, Phys. Lett. B 522, 215 (2001); Phys. Rev. D66, 063501 (2002); D. H. Lyth, C. Ungarelli, and D. Wands, astro-ph/0208055; K. Dimpopoulos and D. H. Lyth, astro-ph/0209180.

5. J. M. Bardeen, P. J. Steinhardt, and M. S. Turner, Phys. Rev. D28, 679 (1983). This quantity was re-introduced by D. Wands, K. A. Malik, D. H. Lyth, and A. R. Liddle, Phys. Rev. D62, 043527 (2000).

6. See, e. g., C. Gordon, D. Wands, B. A. Bassett, and R. Maartens, Phys. Rev. D63, 023506 (2000), Eq. (14). 
7. A. Guth and S-Y. Pi, Phys. Rev. Lett. 49, 1110 (1982).

8. These are taken from Eqs. (15.10.50), (15.10.51), and (15.10.53) of S. Weinberg, Gravitation and Cosmology - Principles and Applications of the General Theory of Relativity (Wiley, New York, 1972). It should be noted that the velocity vector $\mathbf{U}_{1}$ used in this reference has components $U_{1}^{i}=a^{-2} \delta u_{i}=a^{-2} i q_{i} \delta u^{(S)}$.

9. S. Weinberg, Phys. Rev. D64, 123511 (2001); Phys. Rev. D64, 123512 (2001); Astrophys. J. 581, 810 (2002).

10. See, e.g., V. S. Mukhanov, H. A. Feldman, and R.H. Brandenberger, Physics Reports 215, 203-333 (1992).

11. K. A. Malik and D. Wands, Phys. Rev. D59, 123501 (1999). 